PoS $\quad \begin{aligned} & \text { PROCEEDINGS } \\ & \text { OF SCIENCE }\end{aligned}$

\title{
Generalized Parton Distributions: the experimental status
}

F. Sabatié*, H. Moutarde

CEA Saclay - Irfu/SPhN

E-mail: franck.sabatiedcea.fr, herve.moutardedcea.fr

Sixth International Conference on Quarks and Nuclear Physics, April 16-20, 2012

Ecole Polytechnique, Palaiseau, Paris

\footnotetext{
* Speaker.
} 


\section{Introduction}

Generalized Parton Distributions (GPDs) are physical observables which can provide deep insight about the internal structure of the nucleon and more generally, non-perturbative QCD. They contain the usual parton distribution functions (PDFs) and elastic form factors (FFs) as limiting cases or sum rules. In addition, GPDs allow to probe the nucleon as a 3-dimensional object, accessing for instance the quark orbital momentum and picturing the nucleon in quantum phase space.

Generalized Parton Distributions can be accessed through deep exclusive processes such as Deeply Virtual Compton Scattering (DVCS) or Deep Virtual Meson Production (DVMP). About

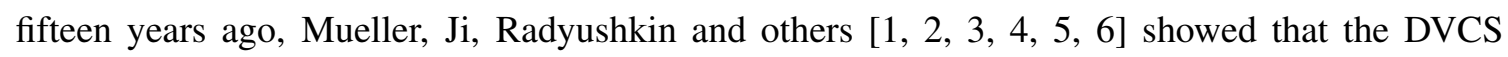
reaction $\gamma^{*} p \rightarrow \gamma p$ can, in the Bjorken limit, be factorized into a hard scattering kernel and a non-perturbative part, containing information about the electromagnetic structure of the nucleon in terms of four twist-2 chiral-even GPDs $H, E, \widetilde{H}$ and $\widetilde{E}$.

These GPDs depend on 4 variables $\left(x, \xi, t ; Q^{2}\right) . x$ characterizes the average light-cone momentum fraction of the struck quark in the intermediate state (not directly accessible experimentally). $\xi$ is the longitudinal momentum fraction of the transfer to the proton $\Delta=p-p^{\prime}$ (where $p$ and $p^{\prime}$ are the initial and recoil proton 4-vectors). Finally, $t=\Delta^{2}$ is the standard Mandelstam variable representing the momentum transfer between the virtual and real photons (or between the target and the recoil proton). The scale evolution of the GPDs ( $Q^{2}$-dependence) has been worked out to next-to-leading order of $\alpha_{S}$ and beyond [ $[$, [8].

In the following, we will introduce the simple case of DVCS to show how this reaction gives access to GPDs. We will then describe a few recent results on DVCS and DVMP from the Jefferson Lab Hall A, CLAS, HERMES and COMPASS collaboration. We will describe the current efforts on GPD extraction and finally conclude on what we have learned so far, as well as what can be expected experimentally in the future.

\section{Deeply Virtual Compton Scattering, a tool to access GPDs}

The photon electroproduction $e p \rightarrow e p \gamma$ can occur either by radiation along one of the electron lines (Bethe-Heitler or BH) or by emission of a real photon by the nucleon (DVCS). The total amplitude $\mathscr{T}_{e p \gamma}$ is therefore the superposition of the BH and DVCS amplitudes:

$$
\left|\mathscr{T}_{\text {ep }}\right|^{2}=\left|\mathscr{T}_{\mathrm{BH}}\right|^{2}+\left|\mathscr{T}_{\mathrm{DVCS}}\right|^{2}+\mathscr{I},
$$

where $\mathscr{T}_{\mathrm{DVCS}}$ and $\mathscr{T}_{\mathrm{BH}}$ are the amplitudes for the DVCS and Bethe-Heitler processes, and $\mathscr{I}$ denotes the interference between these processes.

Using either a polarized beam or a longitudinally polarized target, two separate quantities can be extracted: the difference of cross section for opposite beam helicities or opposite target spin and the total cross section, which at leading twist can be written respectively as:

$$
\begin{aligned}
d \sigma^{\rightarrow}-d \sigma^{\leftarrow} & =2 \cdot \mathscr{T}_{B H} \cdot \operatorname{Im}\left(\mathscr{T}_{D V C S}\right) \\
d \sigma^{\rightarrow}+d \sigma^{\leftarrow} & =\left|\mathscr{T}_{B H}\right|^{2}+2 \cdot \mathscr{T}_{B H} \cdot \operatorname{Re}\left(\mathscr{T}_{D V C S}\right)+\left|T_{D V C S}\right|^{2}
\end{aligned}
$$


where the arrows correspond to the beam helicity. At low beam energy, the pure DVCS contribution is expected to be small with respect to the interference terms, which themselves are in general significantly smaller than the BH term. Note that the DVCS contribution to the difference of cross section only appears at higher twist. It is actually natural to express the DVCS amplitude $\mathscr{T}_{\text {DVCS }}$ at leading twist more generally in terms of so-called Compton Form Factors (CFFs) which can be written at leading order as a function of the GPDs :

$$
\begin{aligned}
\mathscr{F} & =\int_{-1}^{+1} d x F(x, \xi, t)\left(\frac{1}{\xi-x-i \varepsilon}-\frac{1}{\xi+x-i \varepsilon}\right) \quad(F=H \text { or } E) \\
& =\mathscr{P} \int_{-1}^{+1} d x F(x, \xi, t)\left(\frac{1}{\xi-x}-\frac{1}{\xi+x}\right)+i \pi(F(\xi, \xi, t)-F(-\xi, \xi, t))
\end{aligned}
$$

The symbol $\mathscr{P}$ stands for Cauchy principal value. A CFF is complex-valued and we note Re CFF and Im CFF its real and imaginary parts. They are related by fixed- $t$ dispersion relations [Q, 미, ㅁ], ㄴ2], for example :

$$
\operatorname{Re} \mathscr{H}(\xi, t)=2 \mathscr{P} \int_{0}^{1} \frac{d \xi^{\prime}}{\xi^{\prime}} \frac{\operatorname{Im} \mathscr{H}\left(\xi^{\prime}, t\right)}{\frac{\xi^{2}}{\xi^{\prime 2}}-1}+D(t)
$$

where $D(t)$ is a subtraction constant related to the $D$-term [[1]3]. However the $D$-term is poorly known and most of DVCS measurements are made in the region $\xi^{\prime} \leq 0.5$. Using such dispersion relations thus relies on models and may introduce biases in the extraction of GPDs from DVCS data. For that reason the real and imaginary parts of CFFs are taken as independent in some fitting procedures although they should obey the equality ([2.6) from first principles.

\section{Recent experimental results}

Most of the recent experimental results come from DVCS and we will start with that process. The CLAS collaboration is at the final stage of releasing cross sections for photon electroproduction at $6 \mathrm{GeV}$ in a wide kinematical range $0.2<x_{B}<0.6,1<Q^{2}<5 \mathrm{GeV}^{2}$. Along with the older Hall A cross section results [U4], these data will have a significant impact on the GPD extractions and will allow for better constraints on $\operatorname{Im} \mathscr{H}$ and to a lesser extent $\operatorname{Re} \mathscr{H}$. A sample of these preliminary data are shown on Figure $\mathbb{C}$. More information may be found in proceedings from this conference [ए5]].

The CLAS collaboration is also analyzing data on longitudinal Target Spin Asymmetry for DVCS. These data are essential to better constrain the GPD $\widetilde{H}$ through the imaginary part of the corresponding CFF. More information may be found in proceedings from this conference [16].

The Jefferson Lab Hall A collaboration has taken DVCS data recently on both proton and deuteron. The main goal of this experiment is to separate the $\mathrm{DVCS}^{2}$ term from the interference and $B H$ terms in the cross section using a beam energy scan. At HERMES energies, the DVCS ${ }^{2}$ term was evaluated to be small, but at higher- $x_{B}$ and somewhat smaller $Q^{2}$, the conclusion may prove different. More information may be found in these proceedings [[T]].

The HERMES collaboration recently published updated results on beam charge and beam spin asymmetries for DVCS including newly analyzed 2005-2007 data [ए8]. The data from the 19962005 set are compatible with the 2005-2007 sample, and provide about twice the amount of data. A 

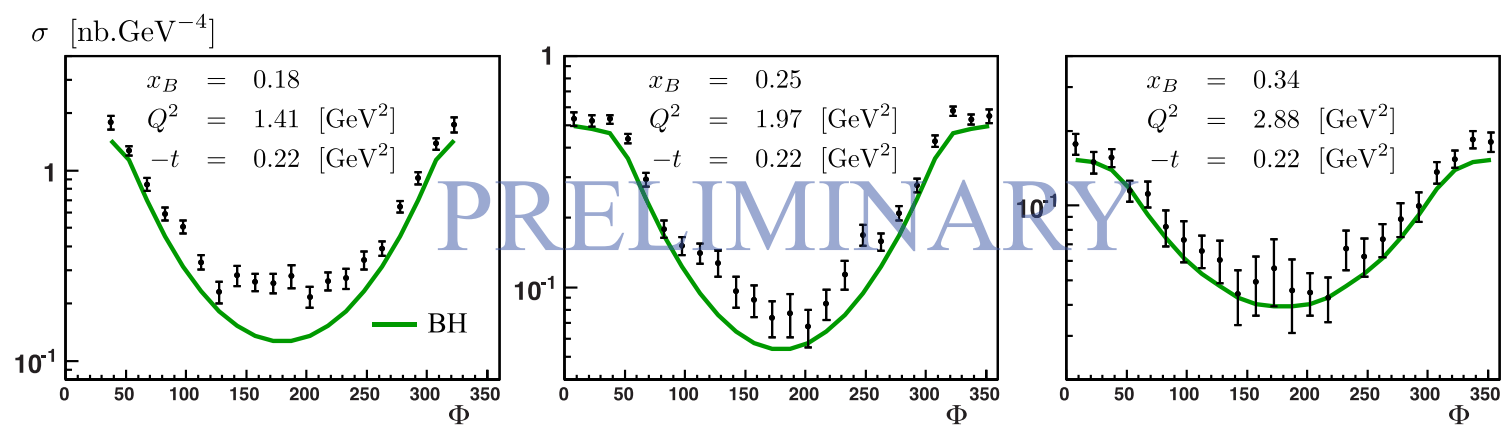

Figure 1: Sample of preliminary CLAS data on photon electroproduction cross sections as a function of $\phi$ for three kinematical bins.
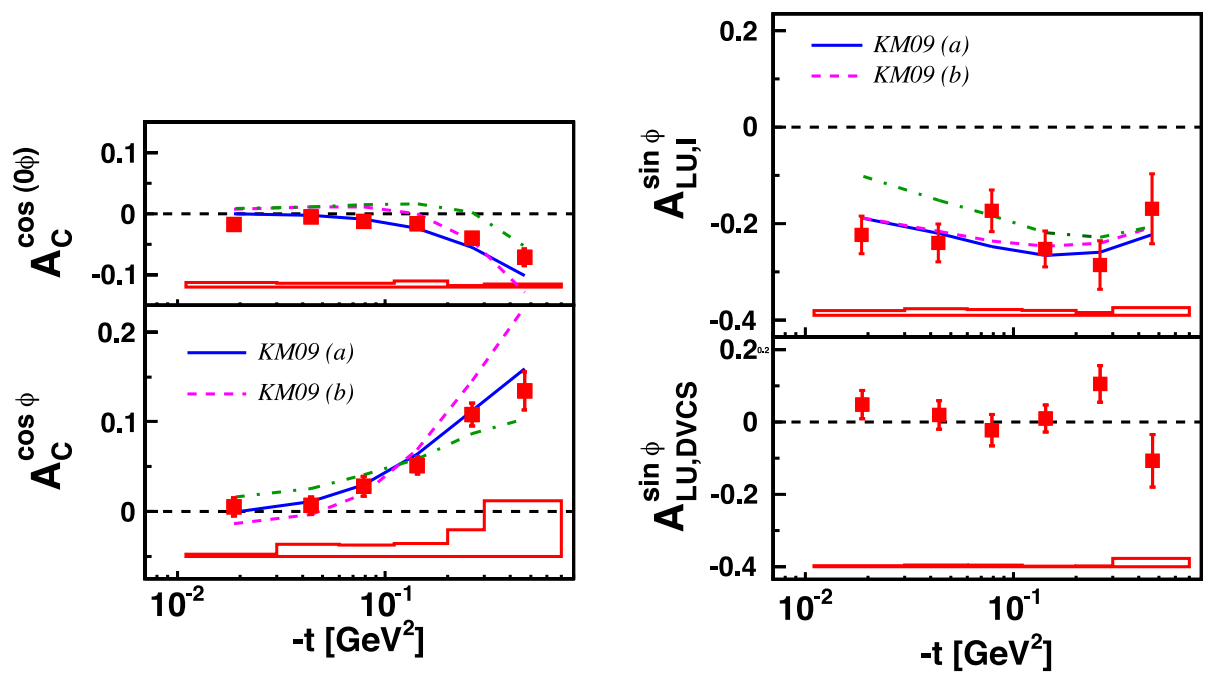

Figure 2: Sample of newly published HERMES data on beam charge and spin asymmetry moments as a function of $-t$, taken from [미].

sample of the most significant moments of the beam charge and beam spin asymmetries published by the HERMES collaboration are shown on Figure 2 . Note that since the time of this conference, the HERMES collaboration released their data on kinematically complete events using their recoil detector. It was interesting to notice a large increase of the beam spin asymmetry in that case, due to the better handling of the dilution coming from inelastic events [एप]].

Concerning meson electroproduction, two recent results are linked to GPD physics: COMPASS finalized its analysis of the $A_{\mathrm{UT}}$ asymmetry for the $\rho^{0}$. More information may be found in these proceedings [ㅁ] ]. CLAS also finalized its analysis of unseparated $\pi^{0}$ electroproduction cross sections []] . They manage to prove that most of the cross section is transverse and that a GPDbased description requires the use of chiral-odd GPDs (also called transversity GPDs). Again, more information may be found in these proceedings [22]. 


\section{Considerations on GPD extraction}

Extractions of GPDs is a much more demanding task than the extraction of Parton Distribution Functions (PDF) or Form Factors (FF) due to the complex functional structures of GPDs. Moreover, we need to extract four functions $H, E, \widetilde{H}$ and $\widetilde{E}$ of three variables $(x, \xi, t)$ for each quark flavour ( $u, d$ and $s)$. The $Q^{2}$-dependence is governed by the QCD evolution equations. Building a flexible yet robust GPD parametrization is a challenging project and the problem is still open today. However, several groups have made attempts to fit GPDs (or CFFs) to data during the last few years. So far, all methods have in common to assume, the dominance of the DVCS twist-2 contribution and are designed to fit several physical observables at once. We will describe the different fitting methods in the following sections.

\subsection{Local fits of CFFs}

The first approach, pioneered in [23] and used in [24, 25, 26, 27] assumes the independence of the real and imaginary parts of CFFs. Each kinematic bin $\left(x_{B}, t, Q^{2}\right)$ is taken independently of the others, and seven values $\operatorname{Re} \mathscr{H}, \operatorname{Im} \mathscr{H}, \operatorname{Re} \mathscr{E}, \operatorname{Im} \mathscr{E}, \operatorname{Re} \widetilde{\mathscr{H}}, \operatorname{Im} \widetilde{\mathscr{H}}$ and $\operatorname{Re} \widetilde{\mathscr{E}}$ are extracted simultaneously. This method has the clear advantage of being almost model-independent. The drawback is that it does not give information on the extrapolation of the extracted CFF outside the data region. In the following we will refer to these fits as local fits.

\subsection{Global fits of GPDs}

In the spirit of the work done on PDFs and FFs, global fits require a physically motivated parametrization of GPDs and deal with all kinematic bins at once. The main advantage is obvious: the ability to extrapolate outside of the data region, and therefore evaluate for instance Ji's sum rule $(t \rightarrow 0)$ or more generally, study the 3D partonic structure of the nucleon $(\xi \rightarrow 0)$. The free coefficients entering the expressions for GPDs are determined either from PDFs and FFs or from DVCS data. Two such studies have been reported recently for DVCS [28, 22] . Note that fixed- $t$ dispersion relations are used as a key ingredient in [28].

\subsection{Hybrid fits of GPDs}

The hybrid fitting procedure used in [24] is a combination of the previous two methods and has been applied with the main assumption of $H$-dominance and twist-2 accuracy. It involves a parametrization which fulfills the polynomiality condition of GPDs and includes $Q^{2}$ evolution at leading order in $\alpha_{S}$. Since this function is otherwise arbitrary, its specific form is a posteriori validated by the quality of the fit. It makes it hazardous to extrapolate the extracted GPD outside the fitting domain as unphysical oscillations may occur. The model dependence is tested by a systematic comparison to local fits and an estimate of the systematic error induced by the $\mathrm{H}$ dominance hypothesis. The good agreement of the local fits with respect to the global fits is a strong consistency check of this approach.

\subsection{Neural network fits of GPDs}

Neural network fits had been successfully performed for PDFs but their use for GPD extraction is quite recent. First results are described in [B] ] within the $H$-dominance assumption. Although it 
is too early to assess the advantages and shortcomings of this approach, it is worth noting that it is a new development in the field of GPD extraction.

\section{Outlook and conclusion}

A sizeable data set is now available from H1, ZEUS, HERMES, Jefferson Lab CLAS, Hall A and COMPASS. The first step of GPD extraction and comparison to models [B] allowed to draw a few conclusions:

- $H$ dominance is not as clear as usually advertised for DVCS in the mid to high- $x_{B}$ region.

- There is a necessity to go beyond simple leading-twist and leading-order formalism.

- That being said, we have a reasonable idea of the size of $H$ for gluons, sea and valence quarks.

- We have a rough idea of the size of $\widetilde{H}$ and $E$ for valence quarks

- We have some limited clues on the size of $\widetilde{H}$ and $E$ for sea quarks.

- We know almost nothing on $\widetilde{E}$ and the chiral-odd GPDs, but there is some progress.

The next stage is obvious : going from a rough to a good knowledge of the GPDs and finally get some insights about the structure of the nucleon. Clearly, accurate data on polarized cross sections in a large kinematical domain are absolutely crucial for that. Advances in theory and phenomenology are also needed, even though projects in GPD extraction have emerged recently with the wealth of new data. In the near and longer term future, we expect even more data to come:

- Short-term (2012-2013) : The CLAS collaboration still has a lot of potential with the cross section data as well as target spin asymmetries soon to be released. The Hall A collaboration is currently analyzing separated DVCS and $\pi^{0}$ data.

- Mid-term (2014-2020+) : The COMPASS-II proposal was approved and a short DVCS run is planned for 2012, with a long run as soon as 2015. The CLAS12 and Hall A collaborations of Jefferson Lab have a huge DVCS and DVMP program including target and beam polarization observables. Note that an exciting new program on Timelike Compton Scattering was just accepted by the Jefferson Lab PAC [B2]].

- Long-term (2025+) : An Electron Ion collider would be the ultimate tool for 3D nucleon imaging with a kinematical reach at high luminosity which would be unparalleled [33]].

\section{References}

[1] D. Mueller, D. Robaschik, B. Geyer, F. M. Dittes and J. Horejsi, Fortsch. Phys. 42, 101 (1994) [hep-ph/9812448].

[2] X. -D. Ji, Phys. Rev. D 55, 7114 (1997) [hep-ph/9609381].

[3] X. -D. Ji, Phys. Rev. Lett. 78, 610 (1997) [hep-ph/9603249]. 
[4] X. -D. Ji, W. Melnitchouk and X. Song, Phys. Rev. D 56, 5511 (1997) [hep-ph/9702379].

[5] A. V. Radyushkin, Phys. Lett. B 380, 417 (1996) [hep-ph/9604317].

[6] A. V. Radyushkin, Phys. Rev. D 56, 5524 (1997) [hep-ph/9704207].

[7] D. Mueller, Phys. Lett. B 634, 227 (2006) [hep-ph/0510109].

[8] K. Kumericki, D. Mueller, K. Passek-Kumericki and A. Schafer, Phys. Lett. B 648, 186 (2007) [hep-ph/0605237].

[9] O. V. Teryaev, hep-ph/0510031.

[10] I. V. Anikin and O. V. Teryaev, Fizika B 17, 151 (2008) [arXiv:0710.4211 [hep-ph]].

[11] I. V. Anikin and O. V. Teryaev, Phys. Rev. D 76, 056007 (2007) [arXiv:0704.2185 [hep-ph]].

[12] M. Diehl and D. Y. .Ivanov, Eur. Phys. J. C 52, 919 (2007) [arXiv:0707.0351 [hep-ph]].

[13] M. V. Polyakov and C. Weiss, Phys. Rev. D 60, 114017 (1999) [hep-ph/9902451].

[14] C. Muñoz Camacho et al, Phys. Rev. Lett 97, 262002 (2006) [nucl-ex/0607029].

[15] H.-S. Jo, these proceedings.

[16] S. Niccolai, these proceedings.

[17] J. Roche, these proceedings.

[18] A. Airapetian et al. [HERMES Collaboration], JHEP 1207, 032 (2012) [arXiv:1203.6287 [hep-ex]].

[19] A. Airapetian et al. [HERMES Collaboration], arXiv:1206.5683 [hep-ex].

[20] K. Schmidt, these proceedings.

[21] I. Bedlinskiy et al. [CLAS Collaboration], arXiv:1206.6355 [hep-ex].

[22] P. Kroll, these proceedings.

[23] M. Guidal, Eur. Phys. J. A 37, 319 (2008) [Erratum-ibid. A 40, 119 (2009)] [arXiv:0807.2355 [hep-ph]].

[24] H. Moutarde, Phys. Rev. D 79, 094021 (2009) [arXiv:0904.1648 [hep-ph]].

[25] M. Guidal and H. Moutarde, Eur. Phys. J. A 42, 71 (2009) [arXiv:0905.1220 [hep-ph]].

[26] M. Guidal, Phys. Lett. B 689, 156 (2010) [arXiv:1003.0307 [hep-ph]].

[27] M. Guidal, Phys. Lett. B 693, 17 (2010) [arXiv:1005.4922 [hep-ph]].

[28] K. Kumericki and D. Mueller, Nucl. Phys. B 841, 1 (2010) [arXiv:0904.0458 [hep-ph]].

[29] G. R. Goldstein, J. O. Hernandez and S. Liuti, Phys. Rev. D 84, 034007 (2011) [arXiv:1012.3776 [hep-ph]].

[30] K. Kumericki, D. Muller and A. Schafer, JHEP 1107, 073 (2011) [arXiv:1106.2808 [hep-ph]].

[31] P Kroll, H. Moutarde and F. Sabatié, article in preparation.

[32] B. Pire, L. Szymanowski and J. Wagner, these proceedings.

[33] R. Ent, these proceedings. 